\title{
EMAIL-BASED INTEROPERABILITY SERVICE UTILITIES FOR COOPERATIVE SMALL AND MEDIUM ENTERPRISES
}

\author{
Hong-Linh Truong ${ }^{1}$, Enrico Morten ${ }^{2}$, Michal Laclavik ${ }^{3}$, Thomas Burkhart ${ }^{4}$, Martin Carpenter ${ }^{5}$, \\ Christoph Dorn ${ }^{1}$, Panagiotis Gkouvas ${ }^{6}$, Kalaboukas Konstantinos ${ }^{6}$, Dario Luiz Lopez ${ }^{7}$, Cesar Marin ${ }^{5}$, \\ Christian Melchiorre $^{2}$, Ana Pinuela ${ }^{7}$, Martin Seleng ${ }^{3}$, Dirk Werth ${ }^{4}$ \\ ${ }^{1}$ Vienna University of Technology, \{truong,dorn $\} @$ infosys.tuwien.ac.at \\ ${ }^{2}$ Softeco Sismat SpA, Italy, \{enrico.morten,christian.melchiorre\}@ softeco.it \\ ${ }^{3}$ Institute of Informatics, Slovak Academy of Sciences, \{laclavik.ui,martin.seleng $\} @$ savba.sk \\ ${ }^{4}$ DFKI, \{Thomas.Borkhart,Dirk.Werth\}@iwi.dfki.de \\ ${ }^{5}$ University of Manchester, \{Martin.Capenter,Cesar.Marin\}@manchester.ac.uk \\ ${ }^{6}$ SingularLogic SA, kkalaboukas@singularlogic.eu \\ ${ }^{3}$ Atos Origin, \{dario.ruiz,ana.pinuela $\} @$ atosorigin.com
}

Keywords: Email, interoperability service utility, SME cooperation, recommendation

Abstract: $\quad$ As most SMEs utilize email for conducting business, email-based interoperability solutions for SMEs can have a profound effect on their business. This paper presents a utility-like system to support specialized SMEs to improve their business via emails by providing system, semantic and process interoperability solutions for individual SMEs and network of cooperative SMEs. We describe the concept of Email-based Interoperability Service Utility (EISU) and a software framework that provides almost zero cost interoperability solutions.

\section{Introduction}

Today's individual, specialized SMEs (small and medium enterprises) are seeking solutions that enable them to cooperate with each other and to establish a network of cooperative SMEs through which they can offer complementary services (see SMEs and cooperation issues in http://ec.europa. eu/enterprise/enterprise_policy/analysis/ doc/smes_observatory_2003_report5_en.pdf ) . Being able to perform cooperative business with other SMEs is crucial to an SME but there are many obstacles preventing SMEs doing so. For example, within (cooperative) SMEs, different types of documents and requests are exchanged, diverse types of flexible and adaptive business processes are executed, and various legacy systems need to be supported; all are related to the interoperability of software systems, semantic documents and business processes between SMEs at different levels. However, SMEs cannot afford to have skilled IT to manage a complex network of SMEs or to take a long learning curve to master complex software. While there exist techniques and framework addressing enterprise interoperabilities at systems, data and process levels, many of them are not suitable for SMEs due to various factors, such as too heavy and costly. SMEs need simple, almost zero-cost solutions.

The lack of almost zero-cost and simple, yet powerful, solutions for SMEs to deal with SME interoperability and cooperation motivates our work in the Commius project (http://www.commius.eu ). Our approach is to provide SMEs with a utility-like capability that guarantees a certain set of common rules for conducting businesses and that is accessible to the SMEs at a very low cost. To implement the utilitylike capability, we rely on email communications by providing an open, flexible software architecture. The rationale is that email systems, based on SMTP and with diverse email clients available, are widely used in most SMEs (see http://www. mariosalexandrou.com/technology-trends/ 2003/80-percent-of-users-prefer-email.

asp) and are Internet-scale. This paper presents the Commius's conceptual architecture which is a generic, easy-to-use, modular and extensible software framework for SMEs to perform business cooperation. We have identified major aspects in mapping the ISU (Interoperability Service Utility) vision (http://cordis.europa.eu/ist/ict-ent-net/

ei-roadmap_en.htm ) into the context of the SMEs having email-based businesses by determining basic requirements that ISU-based software for SMEs should fulfil. The Commius architecture is designed as an open, secured and customizable system, supporting networks of cooperative SMEs to perform their daily business based on emails and Web. With this paper, we contribute a novel approach for the 
interoperability for SME through business models and Commius concepts and architecture.

\section{Motivation and Requirements}

We examine a motivating scenario from Fedit (http://en.fedit.com/ ) which integrates 67 Technology Centers in Spain. Technology Centers (TCs) play a crucial role within the Spanish industrial arena: almost $95 \%$ of the Spanish industrial companies are SMEs, and TCs are the main providers of $R \& D$, innovation and state-of-the art services for these SMEs, including $\mathrm{R} \& \mathrm{D}$ projects, industrial services, training, knowledge and know-how, and generation of intellectual properties.

As an example, one of the obstacles in Fedit is that Fedit does not have a central system that includes and manages TCs. Without such a system, it becomes virtually impossible to commercialize (find customers) and globalize (find synergies) the technology and services offered by the network of TCs. Currently, the majority of the queries and demand for technology and services is received via e-mails, and the time consumed in manually handling the e-mail requests is huge. Besides, misunderstanding of what is actually required and what is written in the e-mail makes the system very inefficient. Fedit, therefore, is searching solutions that is able to make the transition from the request (in e-mail) and the delivery (identification of service/technology) seamless, transparent and efficient. Instead of designing a new system, Fedit would benefit from a system enhancing business activities without changing current work style. It expects various tasks to be continued through emails, but with enhanced, suggestive features. Examples of such tasks are an invoice asking for a specific document, a simple reply to requests or surveys made from Fedit, and order to services and hardware suppliers. Such tasks require interoperability solutions spanning from system to data/semantic to process layers and everything is done via emails, thus bringing various motivations to our work. However, due to the liability of business tasks, Fedit expects that found solutions address interoperability issues and make suggestions, while the final decision should be made by Fedit staff.

After studying many other scenarios from the endusers in Italy and Greece, we have collected requirements for a solution aiming to ease SME interoperability and collaboration in the following way:

- Interoperability solutions must support flexible setup and customization that allows SMEs to manage business content, partners and users, security and privacy, external systems and different types of networks of cooperative clusters.

- Cooperations supported by interoperability solutions have to be monitored to ensure necessary information between different enterprises interoperable and interaction is secured and trusted. These interactions cover both business cooperation and system level interactions.

- Interoperability solutions must support flexible business process execution and monitoring through emails. Business processes refer to the core actions done by the SMEs, such as: prepare and send a request for a business order, receive and process a request according with the agreed business process, cooperate to serve a request.

- The solution must be near-zero cost due to the fact that most SMEs have limited technological deployment and maintenance capabilities.

To allow a near-zero cost, proposed solutions should be relied on fundamental infrastructures that SMEs use to carry out their daily business such as emails. The requirements analysis has revealed the need for an open architecture, where interoperability solutions may be installed locally, interfaced to external systems, and configured for a network of SMEs. Furthermore, the main feature of such solutions is to advise people to improve their work, rather than to fully automatically process all requests.

\section{Email-based Interoperability Service Utility}

We have defined the Email-based Interoperability Service Utility (EISU) inspired by the concept of Interoperability Service Utility (ISU) proposed by the IST Enterprise Interoperability Research Roadmap. Our EISU concept has the following properties:

- Accessible in principle by all SMEs: an emailbased solution will be "accessible" by most SMEs as they typically use email to carry out their business. Therefore, we can support the development of user-centric interoperability services on top of email communication suitable to different business models, such as mail server installation in company premises or through an external service provider.

- Scalable and almost zero cost: email communication is Internet-scale and is almost zero cost. Therefore, EISU can support interoperability of cooperative activities performed and documents exchanged via emails (in human readable form). 
EISU is easy-to-use and imposes no modification to the usual SME practices.

- Guaranteed quality: the quality of interoperability solutions is guaranteed by the reuse of the achievements of the other research projects, by the adoption of the available standards and by the EISU approach, based on always available email system.

- Not controlled by single entity: This can be achieved by ensuring that the software produced in EISU will not be owned by a single entity (e.g., a company or an organization). To this end, the EISU core will be released under an open source software and community share-alike license.

- Supporting a network of cooperative enterprises: EISU will enable the cooperation among SMEs, in particular specialized SMEs. SMEs with different skills and cultural backgrounds will gradually approach the interoperability and cooperate with other SMEs in order to create and diffuse interoperability services suitable for their business models.

With the EISU, an SME can improve its competition by adopting business models and strengthening its cooperation with other enterprises. ERP software vendors may develop EISU modules to support interoperability within their platforms. E-business providers may make available EISU modules to support interoperability with their clients. Our EISU is a novel contribution to the business models for the interoperability for SMEs.

\section{Architectural Overview of Commius}

Figure 1 presents an overview of the Commius architecture which realizes the EISU concept. User tools include tools supporting the user to perform business tasks and to configure Commius. Commius user tools rely on existing email tools and Web browsers and do not require any modification or plugins for email tools and Web browsers. Email Gateway Plugin is responsible for intercepting and postprocessing emails relevant to businesses. Commius Modules and Module Management include systemand business-related modules that handle interoperability tasks to fulfil the request of emails passed through the Commius. Modules in Commius are built from a rich set of components.

System Interoperability components support functionalities for achieving system interoperability, such as providing a basic interoperability infrastructure over SMTP to extract information from emails and annotate emails with new information and to integrate Commius with external systems in order to access legacy/external information and services. Semantic Interoperability components include components providing functionalities for achieving semantic interoperability, such as providing facilities to achieve semantic alignment, facilitate concept negotiation, and to annotate messages with meta-data to embed semantics within them. Process Interoperability components offer functionalities for supporting process interoperability, such as features to configure process modules, to match and adapt business processes. Data Management components include components providing data management facilities which are needed by Commius. Security and Privacy components include components providing security and privacy infrastructure for Commius. Hosting environment is the execution environment which hosts Commius components, modules and services. The core entities of the Commius can interact with external systems, which are not part of the Commius. External systems include common/specific legacy systems in SMEs as well as other services supporting the business of SMEs.

The architectural overview in Figure 1 describes a single Commius system which can be deployed for a single SME. Such a deployment can be done by the SME itself or by a Commius service provider under the SaaS model. Individual Commius systems can be connected to establish a cooperative network of Commiuss to support a network of SMEs. The key point of our open system is that interoperability solutions are implemented in different components. Then, we develop software modules to compose components to offer utilities required by SMEs. Our system is extensible in the sense that different components and modules can be built and added into Commius by different developers and providers. Through module description and email extraction a process is used to find suitable modules for processing emails and the results produced by modules will be composed to produce suggestions to the user. Commius design is our contribution to a software architecture for addressing intereropability among SMEs via emails. We discuss some main aspects of Commius architecture in the following sections.

\subsection{Matching Emails to Modules}

When an email arrives, the Email Gateway Plugin will extract metadata from the email and its attachments, such as sender and receiver ids, existing Com- 


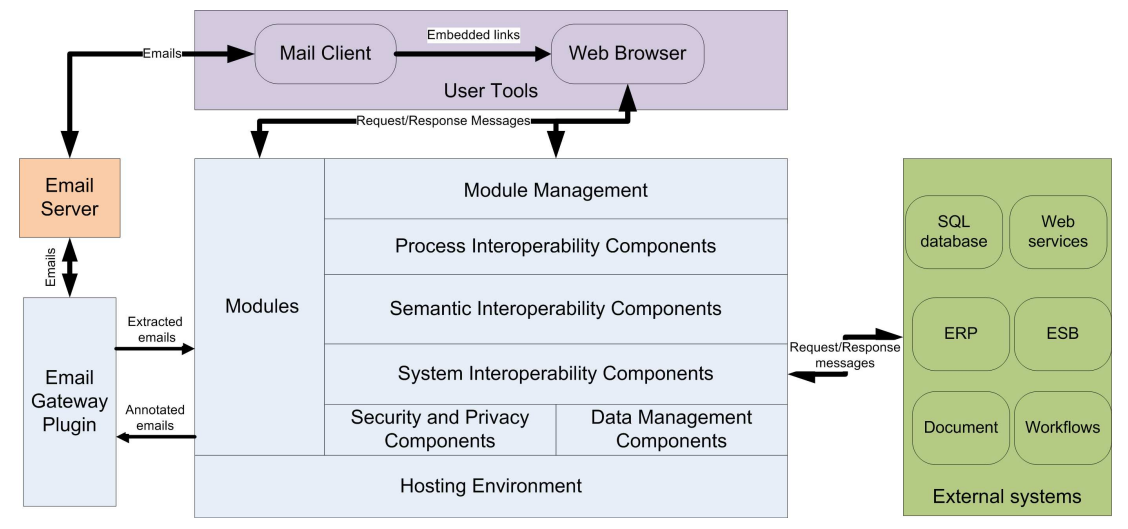

Figure 1: Architectural overview of Email-based Interoperability Service Utility for SMEs

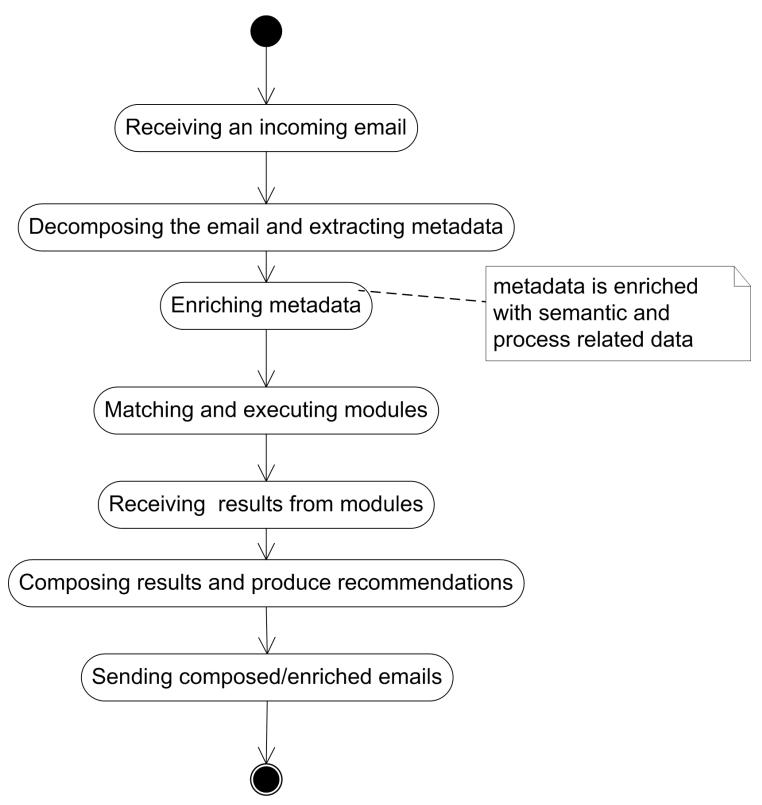

Figure 2: Overview of handling emails in Commius

mius embedded header information, and relevant keywords. The extraction is performed by applying regular expression rules which are predefined and updated regularly. The extracted metadata, a set of keys and values, is then enriched with other metadata related to business activities. This enrichment is achieved by using semantic and process interoperability components to analyze the extracted metadata.

Based on the enriched metadata, the Module Manager performs a matching process to select the right module to handle the email. This matching process utilizes various sources of information, including extracted keywords, module description, historical data, rules, and components, and in particular, semantic information. For example, based on pre-defined rules and metadata a module can be selected. This happens when the user knows for sure which modules should process which emails. When selecting a module, the Module Manager can also interact with the user, in case it cannot decide the right module (e.g., due to rule conflict or missing information). In this case, the Module Manager will inform the user with an email including embedded links and by relying the email or clicking the links, a module can be selected.

When a module is identified, the email and enriched metadata are forwarded to the module which processes the request (see Section 4.2). Here, in the matching process, the Module Manager treats modules as black boxes. Then it obtains the results from the modules and passes the result to the Email Gateway Plugin which combines and prepares the result in a right format before sending the resulting email(s) to appropriate recipients.

\subsection{Business Modules and Module Development}

Business modules implement specific tasks in the context of a defined set of business processes within a determined business domain. These modules consist of a set of software components to provide the business support as well as of a set of configuration data that sets up the components in order to operate accordingly to the process suitable for domain needs. A key element of our architecture is the methodology to develop business modules which implement business solutions. Because a business module typically implements specific business processes of SMEs, we need to provide generic, yet simple, mechanism to develop, manage and share business modules. In our architecture, business modules have well-defined interface and description provided by their developers. Business modules utilize system, semantic and process components to process emails not only based on metadata but also based on business process template/configuration. Modules can be deployed and ex- 
ecuted on demand through plug-in mechanisms.

Modules may also be exposed as Web services, thus allowing them to be accessed through the Web. This allows us to support different types of interactions with the user of Commius. There are many tasks the user will conduct via emails, which a module can handle by processing emails, and there are tasks where the user can simply interact with the module through a Web browser, for example, to confirm a step while the module handling the request.

The advantage of using modules is that we support both the end user, the developer, and the provider to use and build their solutions. For example, the provider can implement a module and specify module description and business process templates guiding the operation of the module; or the developer can simply use an existing module but provide new business process templates. The end user may just need to obtain modules and change module configuration and business process templates suitable for his/her environments. Thus, business objectives are achieved through the implementation of modules and/or the configuration of business process templates.

\subsection{User Interactions}

For SMEs end users, easy-to-use tools are critical. First, we assist the user to select suitable modules to execute requests coming from emails. This includes the selection of the right modules, the configuration of the interface between Commius and user's external systems, and the sematic mapping of business documents. Second, we provide tools to assist the endusers to perform their business carried out by business modules. Examples of supporting tools for assisting the end-user to perform their business are a Task Recommendation Tool to recommend relevant human tasks in a process and provide extra information for a task, and a Task Tracking Tool allowing a user to track performed steps in a running/finished process and to directly embed user history or useful process information into an Commius enhanced email.

The key benefit that Commius brings to the users is to support them to simplify their business by enriching emails with hints and recommendations without any change made in mail clients or Web browsers. By exploiting system, semantic and process interoperabilities features, Commius enriches emails with business-related information, such as preparing templates for order confirmation, tracking information, and payments. Enriched information is embedded into emails that can be accessed via external links.

\section{Support SME Cooperation}

The Commius also supports a network of cooperative SMEs by extending individual Commius systems to a trusted network of cooperative SMEs. Within a network of cooperative Commius-based SMEs, each company utilizes its own Commius system, hosted in its infrastructure or provided by an SaaS provider. Based on agreements in the network, security and privacy policies are established to ensure that a company can send some requests to another company.

\subsection{Semantic Understanding}

As email-based business requests are flexible, we cannot assume that everyone communicating with the company will follow specific formats or indeed that they use Commius. This motivated our proposed peer-to-peer shared documents approach of which the basic idea is to focus on the information within the documents rather than their actual syntax. To do this we use: (i) a set of standardized tokens representing atomic items of information which can be found within a document, (ii) a set of rules for pulling these tokens out of documents, and (iii) a set of local document types defined in terms of the information tokens.

The basic approach is then to take an incoming document, identify what information tokens it contains and hence identify the closest matching local document type(s) and insert the information into these. In the peer-to-peer document sharing, each Commius deployment includes an initial set of information tokens and rules for identifying these. Each company then uses these tokens to define its local document types. When an SME receives a document (e.g., a purchase order) via email it tries to map it to a local document type in order to understand it in the company's own terms. If it cannot map it then the Commius in that company contacts the Commius in the sender company to find a common semantic understanding of the information continaed in the document. Upon agreement, the Commius performs the mapping using possibly a new document type.

\subsection{Cooperative Business Activities}

We develop a peer-to-peer model for cooperative activities that supports companies in the same network to assist each other in answering requests from the user. When a user of an SME sends a request, the SME's Commius system might not be able to solve the request. In this case, the SME can use the cooperative network to find other SMEs which can solve the request. The peer-to-peer model for cooperative 
activities works on the assumption that each Commius deployment includes rules and user interactions for identifying when a request should be forwarded to another SMEs. Such rules are updated over the time based on business exchange within the network and are based on the trust degree among SMEs. Rules can be used to (semi-)automatically forward emails or to create a new request based on an existing one and to ask for approval from the user. Based on specific configuration, an SME receiving a request from another SME in the network might treat the request in a different way with a request from its own user.

\section{Related Work}

Various projects have connected email to knowledge or context-sensitive information, such as Xobni (http://www.xobni.com ), kMail (Schwartz and Teeni, 2000) and Zimbra (http://www.zimbra. com), but they aims at supporting human collaboration, rather than enterprise interoperability. Another major research direction is to address task management in emails, such as visualization of complex corporate collaboration (Wattenberg et al., 2005), recognization of relations between messages (Bellotti et al., 2003), social profiles and network (Whittaker et al., 2004), users' email behavior (Horvitz et al., 1999), and filing messages (Segal and Kephart, 1999). In particular, (Wattenberg et al., 2005) presents lesson learned in using email supports for corporate collaborations, but it does not address cooperative activities among enterprises. There are also various commercial efforts atop email for business, such as collective knowledge gathering in MarkMail (http: //markmail.org/ ), and prioritizing emails in Attent solution from Seriosity (http://www.seriosity. com/attent.html ). However, one major problem is that many of these systems involved the development of new standalone clients - forcing people to switch to new software and change their work practices. A second problem is that these systems were often not well integrated with users' external/legacy systems (e.g., their file system or company databases).

Recently, there is also ongoing research on extracting information and knowledge, its management, social network analysis, and topic discovery from emails, such as in the introduction of the Enterprise track within the series of TREC conferences (http://trec.nist.gov/pubs/ trec14/t14_proceedings.html ) and the Nepomuk project (http://nepomuk. semanticdesktop.org ). However, supports of business interoperability as what Commius intends for SMEs are missing.
Various process interoperability techniques have been developed, such as the projects ATHENA (http://www.modelbased.net/aif/index.html ) and INTEROP (http://interop-vlab.eu ). The results of these projects can be used within Commius to support a business driven visualization of collaborative processes and business process interoperability for SMEs.

\section{Conclusion}

In this paper, we have presented an Email-based Interoperability Service Utility (EISU) for cooperative SMEs. Based on that, we have designed the Commius architecture which includes main components and modules for realizing EISU and discussed possible customization and deployments of Commius for networks of cooperative SMEs. Currently, we utilize existing security protocols. The privacy and business compliance is an ongoing work. The implementation of Commius is currently on progress and we expect to have the first prototype released in Summer 2009.

\section{ACKNOWLEDGEMENTS}

We thank all our colleagues in Commius for their contributions. The research reported in this paper is funded by the EU under the FP7 Commius project.

\section{REFERENCES}

Bellotti, V., Ducheneaut, N., Howard, M., and Smith, I. (2003). Taking email to task: the design and evaluation of a task management centered email tool. In CHI '03: Proceedings of the SIGCHI conference on Human factors in computing systems, pages 345-352, New York, NY, USA. ACM.

Horvitz, E., Jacobs, A., and Hovel, D. (1999). Attentionsensitive alerting. In Laskey, K. B. and Prade, H., editors, UAI, pages 305-313. Morgan Kaufmann.

Schwartz, D. G. and Teeni, D. (2000). Tying knowledge to action with kmail. IEEE Knowledge Management, pages 33-39.

Segal, R. and Kephart, J. O. (1999). Mailcat: An intelligent assistant for organizing e-mail. In Agents, pages 276282

Wattenberg, M., Rohall, S. L., Gruen, D., and Kerr, B. (2005). E-mail research: targeting the enterprise. Hum.-Comput. Interact., 20(1):139-162.

Whittaker, S., Jones, Q., Nardi, B., Creech, M., Terveen, L., Isaacs, E., and Hainsworth, J. (2004). Contactmap: Organizing communication in a social desktop. $A C M$ Trans. Comput.-Hum. Interact., 11(4):445-471. 